\title{
Targeted association and intracellular delivery of nanocargoes into primary T lymphocytes via interleukin-2 receptor-mediated endocytosis
}

\author{
V. S. S. Abhinav Ayyadevara ${ }^{1}$, Armin $\mathrm{Ahmadi}^{2}$, Kyung-Ho Roh ${ }^{1,2, *}$ \\ ${ }^{1}$ Department of Biotechnology Science and Engineering, University of Alabama in Huntsville, \\ Huntsville, Alabama 35899, United States \\ ${ }^{2}$ Department of Chemical and Materials Engineering, University of Alabama in Huntsville, \\ Huntsville, Alabama 35899, United States \\ * To whom correspondence should be addressed:
}

Kyung-Ho Roh, $\mathrm{PhD}$

Assistant Professor

Department of Chemical and Materials Engineering

University of Alabama in Huntsville

301 Sparkman Drive NW

Huntsville, AL 35899

Telephone: 256-824-5292

Email: kyung-ho.roh@uah.edu 


\section{ASSOCIATED CONTENT}

Supplementary Figure 1. Effect of IL2 starvation on the surface expression level of IL2R subunits on Day-2 $\mathrm{T}$ cells.

Supplementary Figure 2. Structure and properties of mouse and human IL2.

Supplementary Figure 3. Potency of murine and human IL2 on mouse T cells.

Supplementary Figure 4. Quenching efficiencies of varying percentages of Trypan Blue for extracellular green fluorescence.

Supplementary Figure 5. Loading conditions for biotinylated lysozyme (model protein) onto the surface of SPIONs.

Supplementary Figure 6. Effects of the presence of free IL2 during incubation on the delivery of SPION50 to Day-3 T cells.

Supplementary Figure 7. Validation for exclusive and complete quenching of extracellular green fluorescence from the SPION50 labelled with Alexa Fluor 488 using 0.4\% trypan blue.

Supplementary Figure 8. Transmission electron microscope (TEM) image of Day-3 T cells incubated with SPION50_7.

Supplementary Figure 9. Mean fluorescence intensity (MFI) analysis for the delivery of IL2loaded SPION50_7, SPION100_2, and SPION200_2 on the T cells over the course of 12-Day culture. 
(a)

(b)
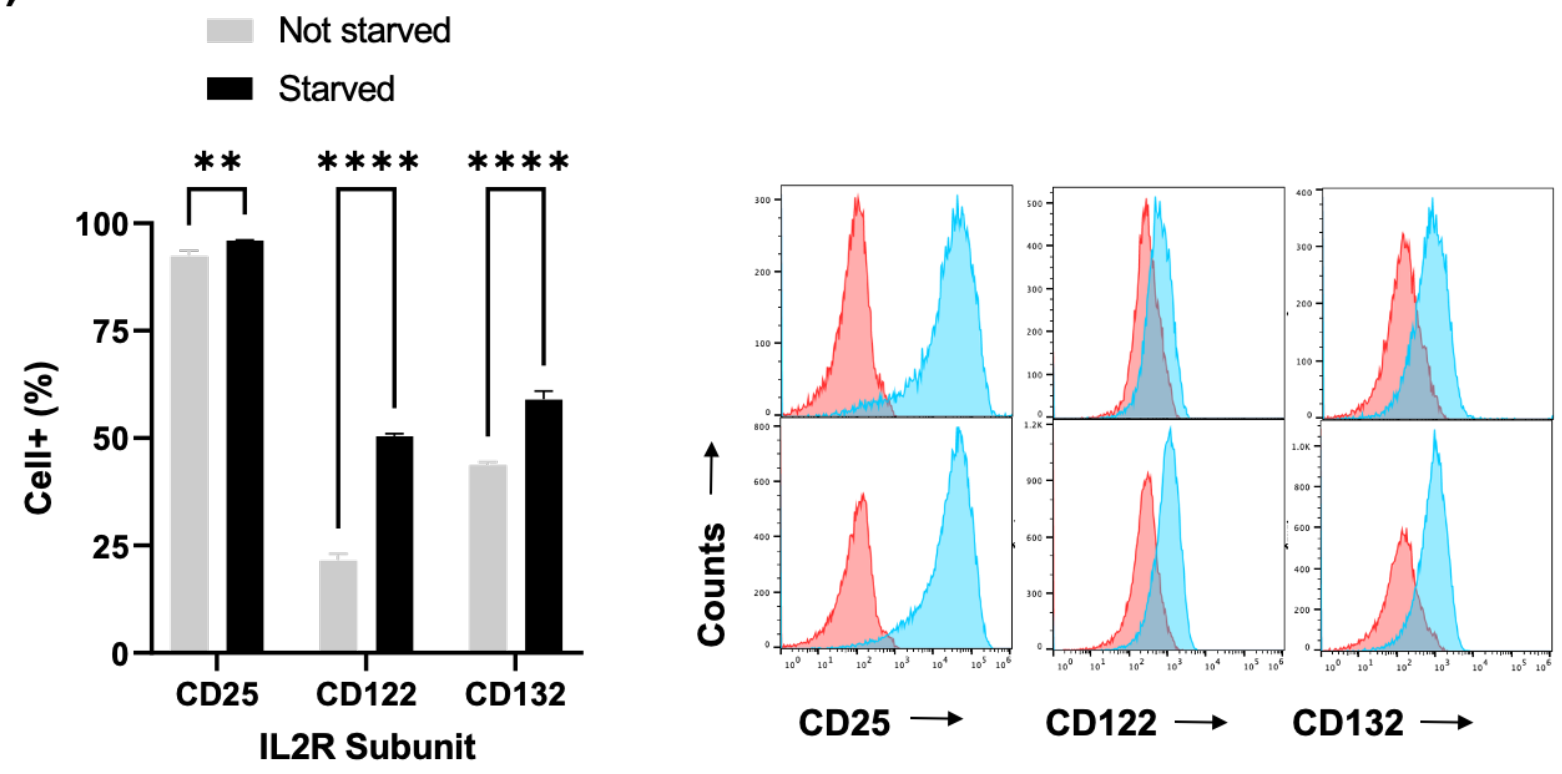

Supplementary Figure 1. Effect of IL2 starvation on the surface expression level of IL2R subunits on Day-2 $T$ cells. Expression levels of indicated subunits were measured by flow cytometry with or without the IL2 starvation for 165 minutes. (a) Percentage positive for indicated IL2R subunits; Results are represented as mean $\pm \mathrm{SD}(\mathrm{n}=3$, two-way ANOVA with Sidak's multiple comparisons, ${ }^{* *} p<0.01$ and ${ }^{* * * *} p<0.0001$ ). (b) Representative raw histograms of "not starved" (top) and "starved" (bottom) conditions; red and blue colors indicate the unstained controls and the stained samples, respectively. 
(a)

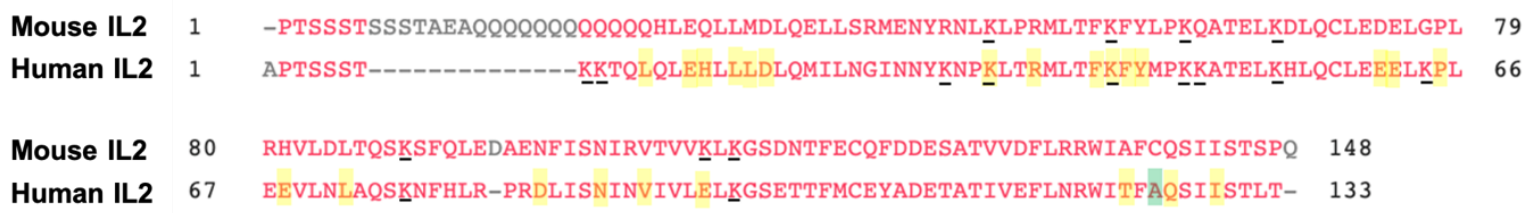

(b)

\begin{tabular}{c|c|c|c|c}
$\begin{array}{c}\text { IL2 } \\
\text { species }\end{array}$ & $\begin{array}{c}\text { Mol. Wt. } \\
\text { (Da) }\end{array}$ & $\begin{array}{c}\text { Theoretical } \\
\text { pl }\end{array}$ & Gravy & $\begin{array}{c}\text { \#Lysine } \\
\text { residues }\end{array}$ \\
\hline Mouse & $17,160.3$ & 4.88 & -0.546 & 07 \\
\hline Human & $15,517.1$ & 6.83 & -0.161 & 11
\end{tabular}

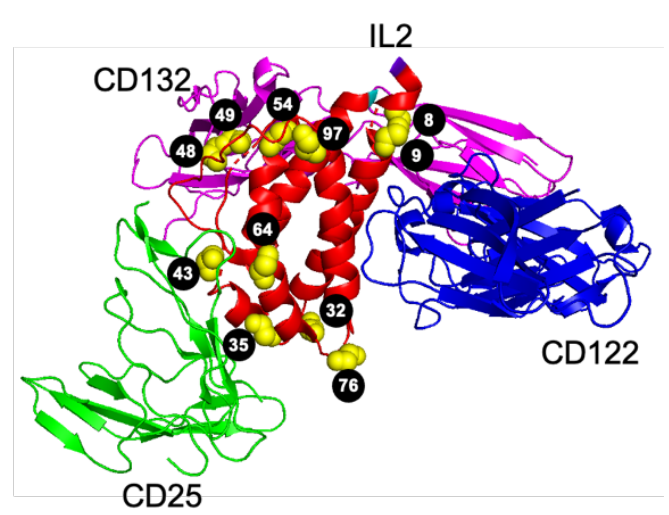

Supplementary Figure 2. Structure and properties of mouse (mIL2) and human IL2 (hIL2). (a) Protein BLAST alignment of mIL2 (P04351) and hIL2 (P60568); Residues in yellow within hIL2 sequence are known to interact with the human IL2R; Lysines are underlined; Cysteine 125 in the original CDS of hIL2 is substituted with Alanine (green) in recombinant hIL2. (b) Properties of recombinant mIL2 and hIL2; "Gravy" refers to Grand Average of Hydropathicity obtained from ProtParam tool in www.expasy.org. (c) A side view of crystal structure of hIL2 (red) complexed with IL2R (PDB ID: 2ERJ) depicted using Pymol; CD25 (green), CD122 (blue), and CD132 (magenta); Lysine residues are represented as yellow spheres with their respective amino acid numbers. T3 and T133 of hIL2 are labelled in purple and cyan, respectively, to aid localization of termini of hIL2 in the crystal structure. 


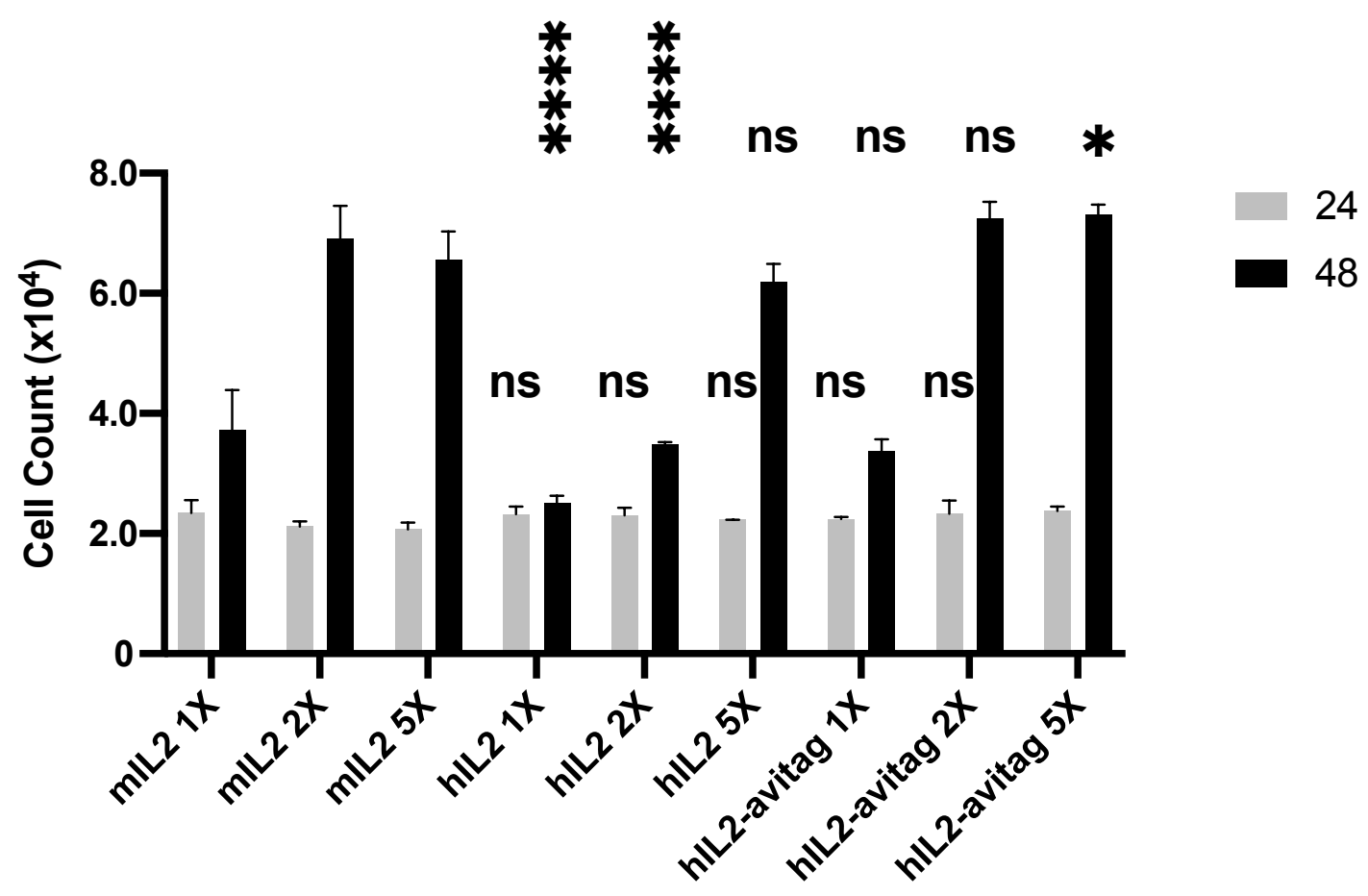

Supplementary Figure 3. Potency of murine and human IL2 on mouse T cells. The potencies of different doses of mIL2, hIL2, and hIL2-Avitag were compared by measuring number of cells after 24- and 48-hour incubation on Day-9 cells of a mouse T cell culture. Each value of hIL2 and hIL2-avitag was compared to the respective mIL2 control and denoted with the corresponding statistical significance marks. Results are represented as mean $\pm \mathrm{SD}(\mathrm{n}=3$, one-way ANOVA with Dunnett's multiple comparisons, $* p<0.05, * * * * p<0.0001)$ 
(a)

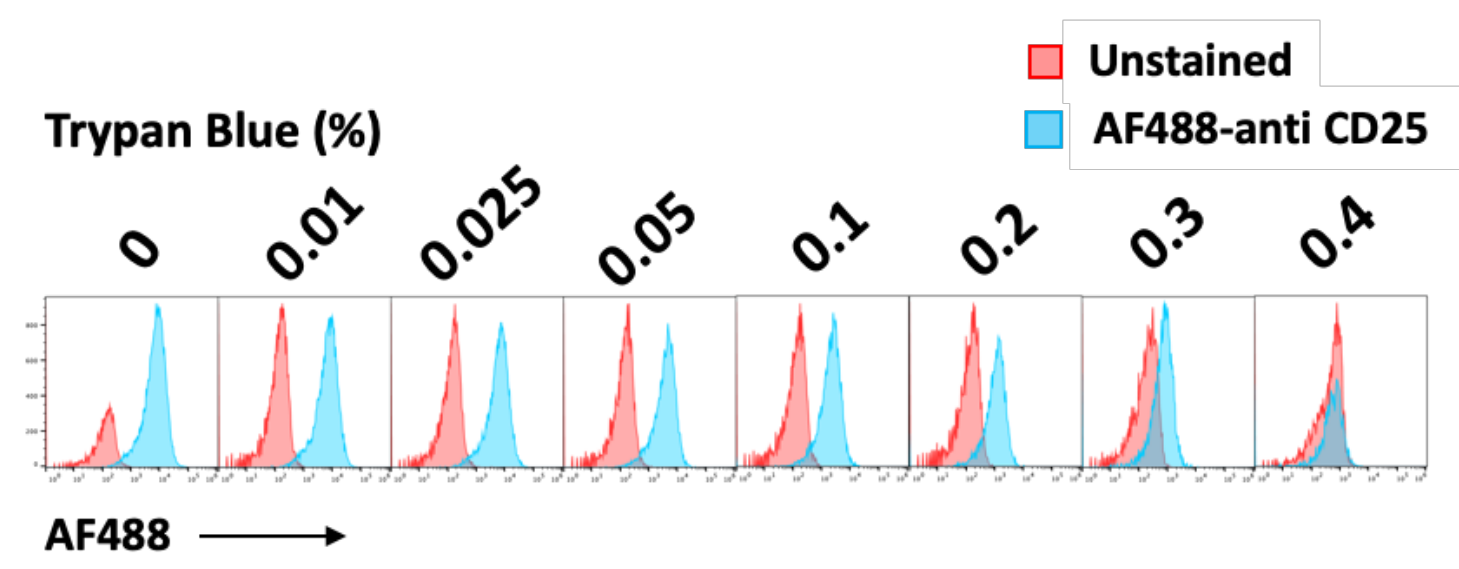

(b)

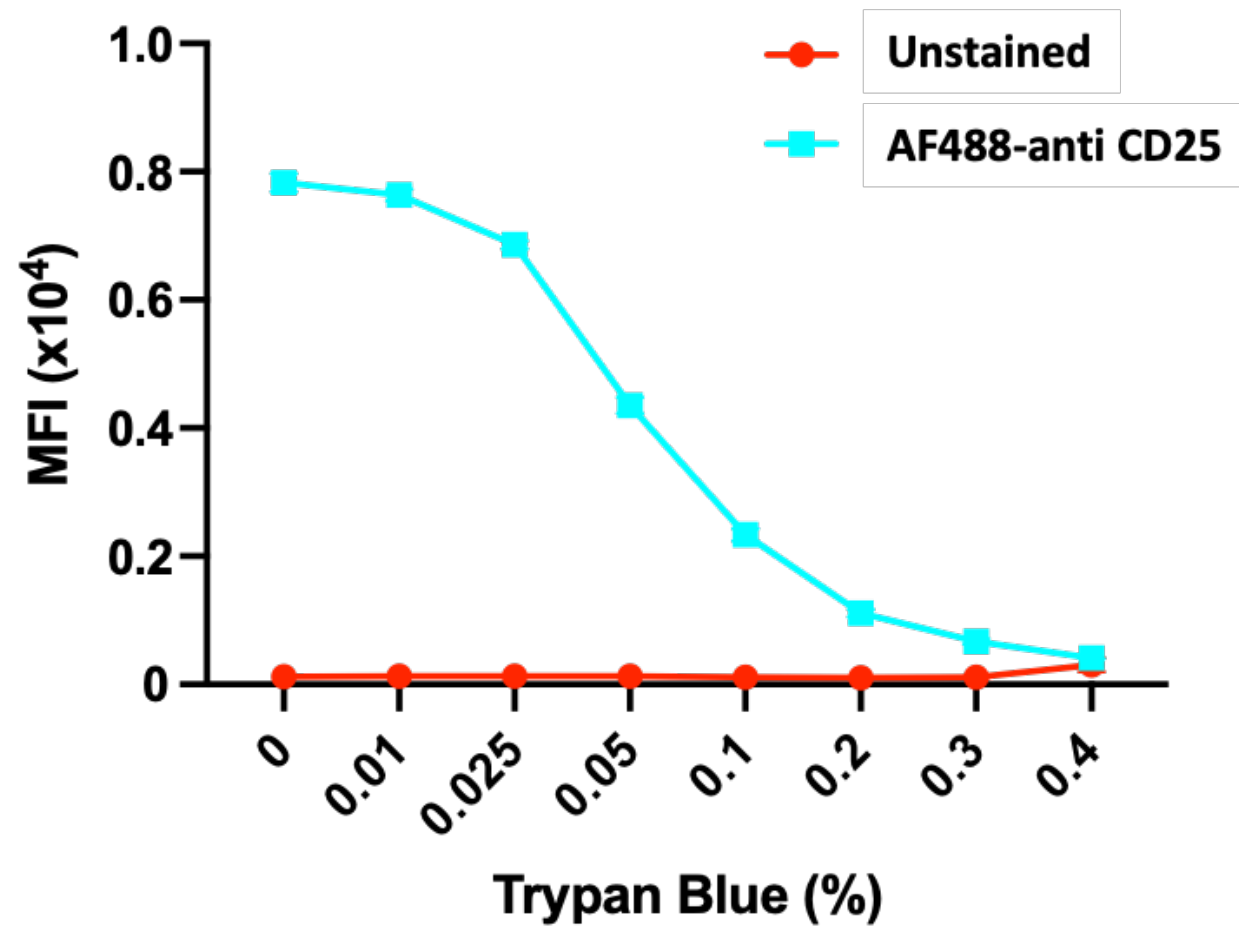

Supplementary Figure 4. Quenching efficiencies of varying percentages of trypan blue for extracellular green fluorescence. For fixation, murine primary pan T cells (on culture Day 2) were incubated within $2 \%$ paraformaldehyde for 30 minutes at room temperature. The fixed $\mathrm{T}$ cells were stained by Alexa Fluor 488-labelled anti-mouse CD25 antibody (clone 7D4) before being analyzed by flow cytometry in the presence of indicated percentage of Trypan Blue. (a) A collection of representative histograms. (b) The mean fluorescent intensity (MFI). Results are represented as mean $\pm \mathrm{SD}(\mathrm{n}=3)$. 
(a)

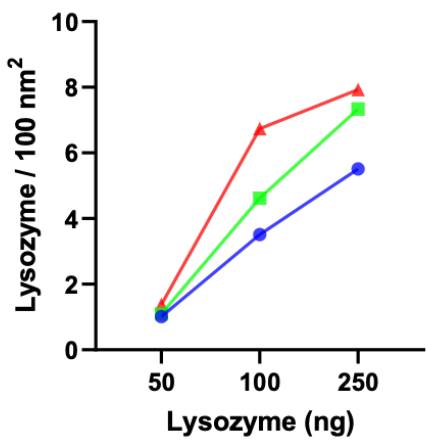

(b)

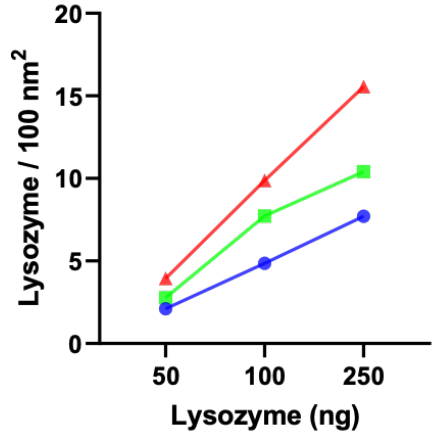

(c)

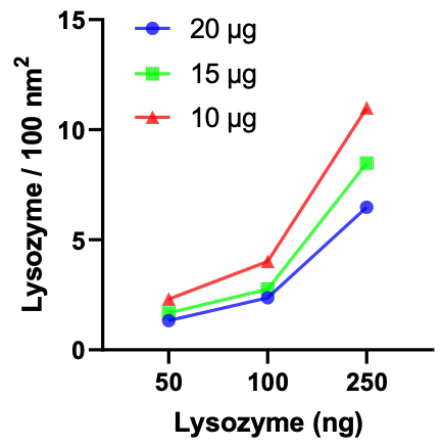

Supplementary Figure 5. Loading conditions for biotinylated lysozyme (model protein) onto the surface of SPIONs. Correlations between the quantity of biotinylated lysozyme and the resulting surface density were made for (a) SPION50 (b) SPION100 and (c) SPION200; Varying amounts, 10, 15, and $20 \mu \mathrm{g}$ of SPIONs were employed in each incubation reaction, which were indicated in red, green and blue color, respectively. 
(a)

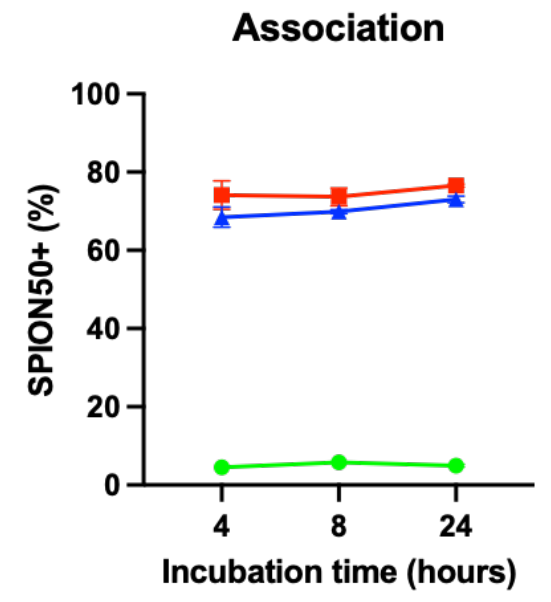

(b)

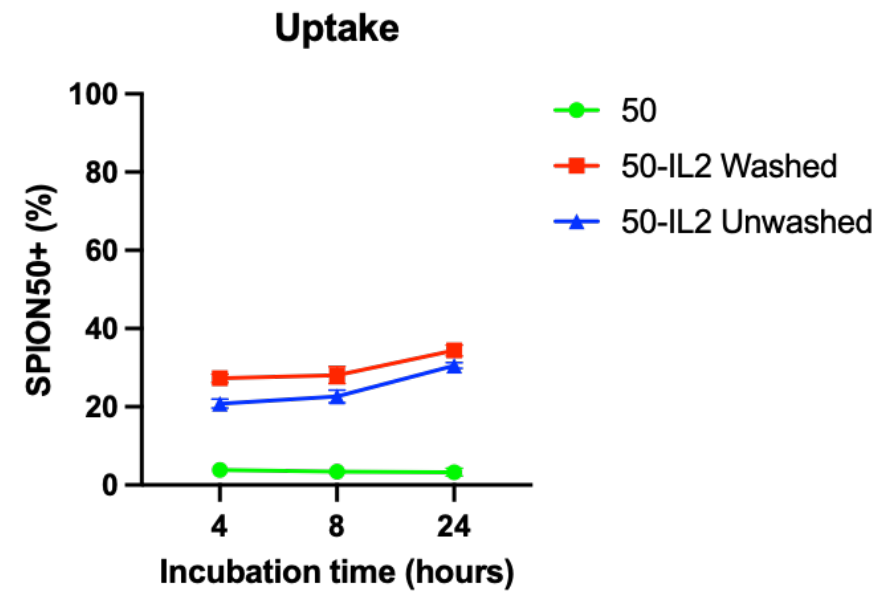

Supplementary Figure 6. Effects of the presence of free IL2 during incubation on the delivery of SPION50 to Day-3 T cells. Alexa Fluor 488-labeled SPION50s were loaded with 345 molar excess of hIL2-Avitag. "50" in green color indicates the SPION50s without loaded hIL2. The association (a) and uptake (b) of hIL2-loaded SPION50s after indicated hours of incubation with or without the free IL2 were measured by flow cytometry, indicated as "50-IL2 Unwashed" in blue and "50-IL2 Washed" in red, respectively. Results are represented as mean \pm SD $(n=3)$. 
(a)

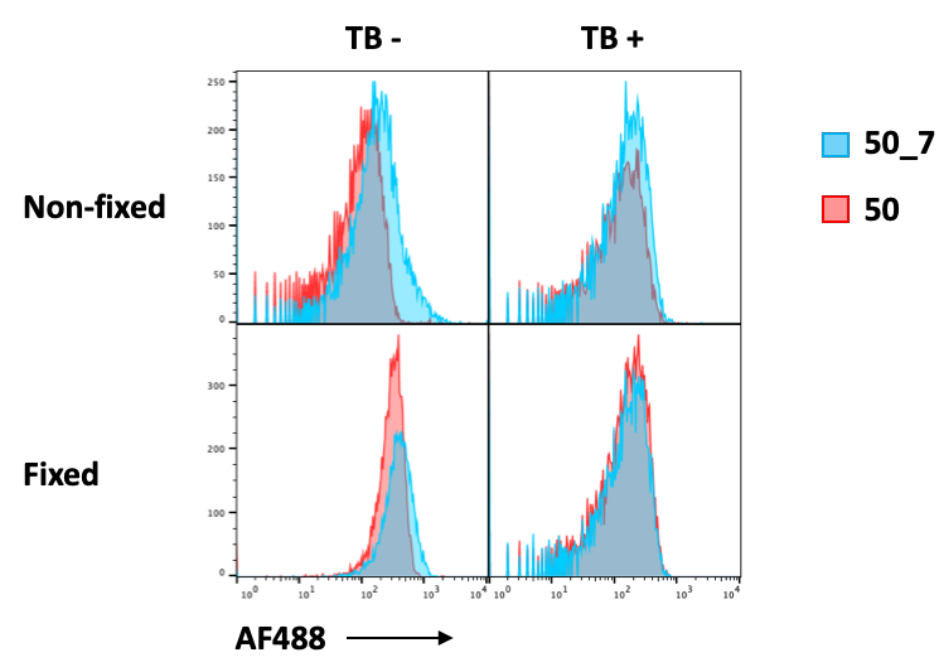

(b)

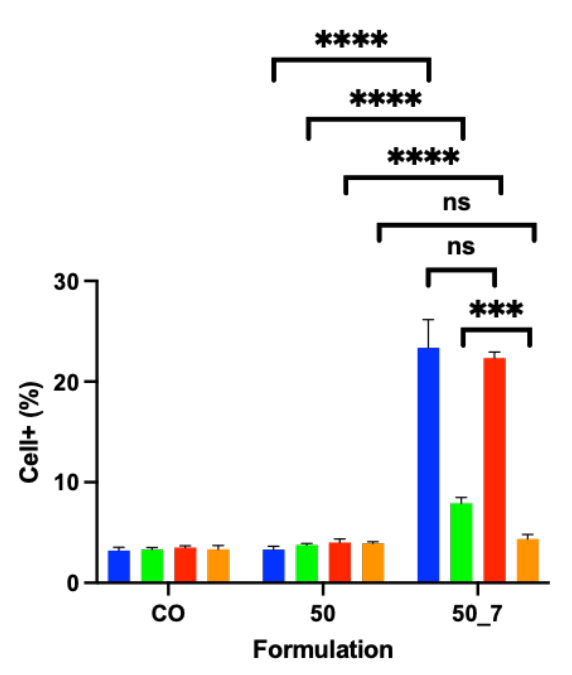

(c)
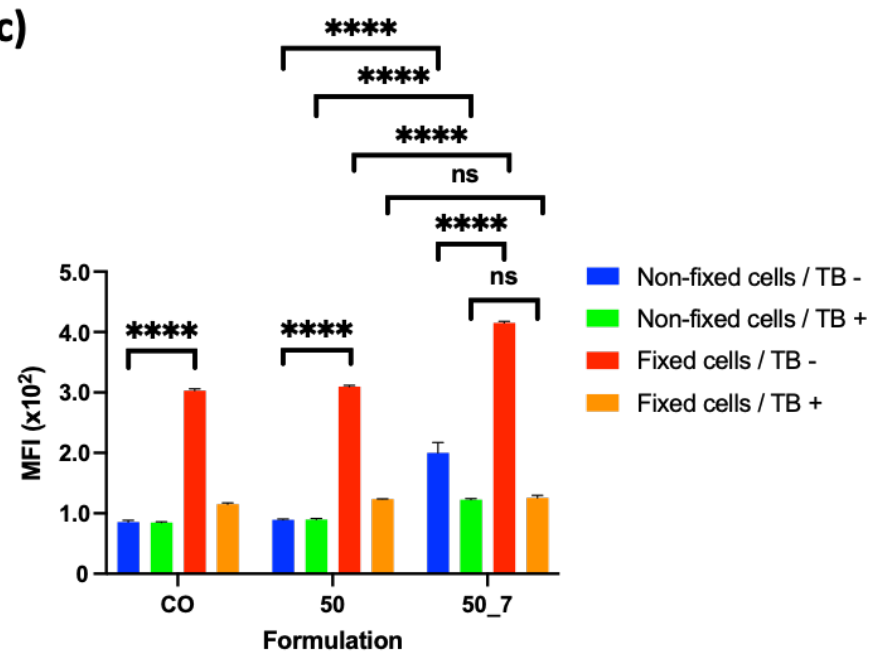

Supplementary Figure 7. Validation for exclusive and complete quenching of extracellular green fluorescence from the SPION50 labelled with Alexa Fluor 488 using $0.4 \%$ trypan blue. Fixed and non-fixed murine pan T cells (on culture Day 3 ) were incubated with indicated SPIONs for 24 hours before being analyzed by flow cytometry. "TB-" and "TB + " indicate the absence and presence of trypan blue during the flow cytometry measurements, respectively. " 50 " and "50_7" indicate the unmodified SPION50 and the SPION50 loaded with IL2 at surface density of 7 IL2 per $100 \mathrm{~nm}^{2}$, respectively. "CO" indicates cell only control group. (a) A collection of representative overlays of histograms for non-fixed $v s$ fixed cells, with or without trypan blue. (b) Percent of AF488-positive cells and (c) mean fluorescence intensity (MFI) of AF488. (b, c) Results are represented as mean $\pm \mathrm{SD}(\mathrm{n}=3$, 2-way ANOVA with Dunnett's multiple comparisons, *** $\left.p=0.0002,{ }^{* * * *} p<0.0001\right)$. 


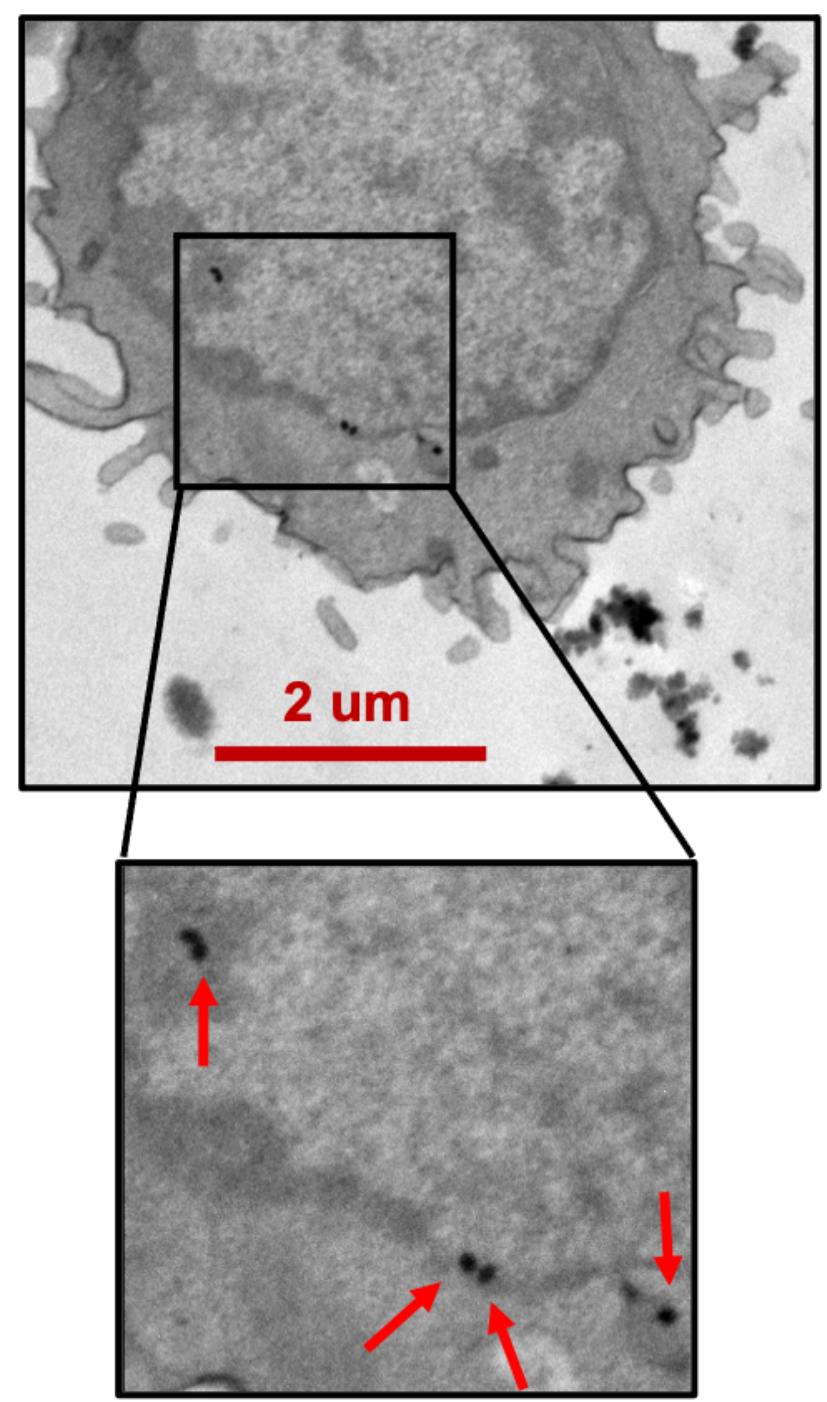

Supplementary Figure 8. Transmission electron microscope (TEM) image of Day-3 T cells incubated with SPION50_7. Red arrows are added to clearly indicate the SPION50s. 
(a)

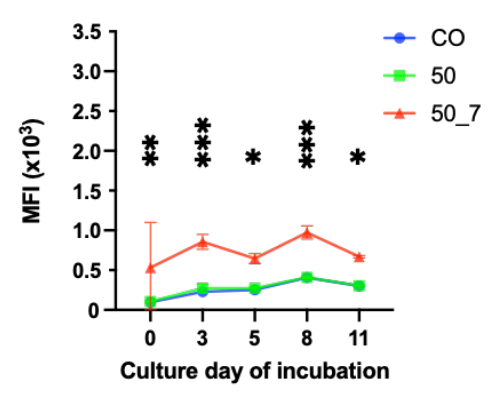

(d)

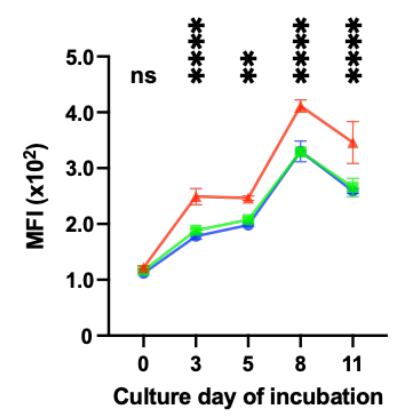

(b)

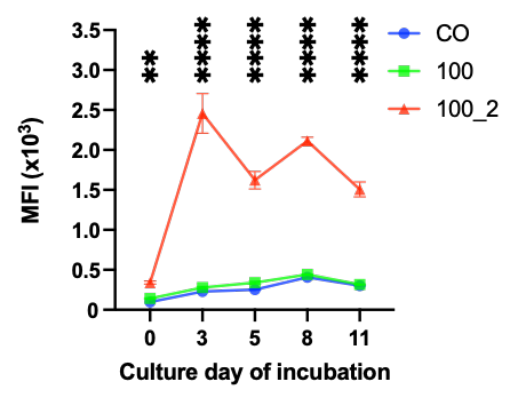

(e)

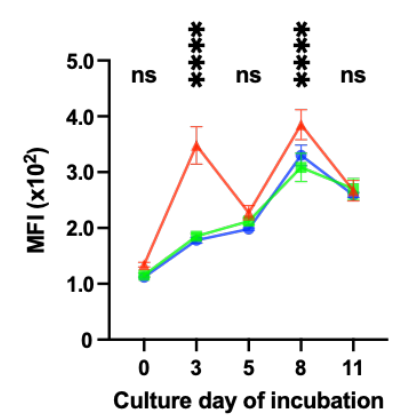

(c)

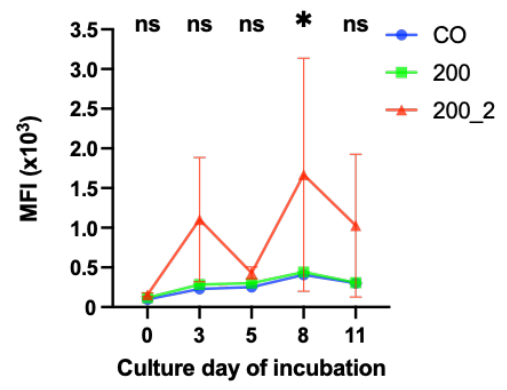

(f)

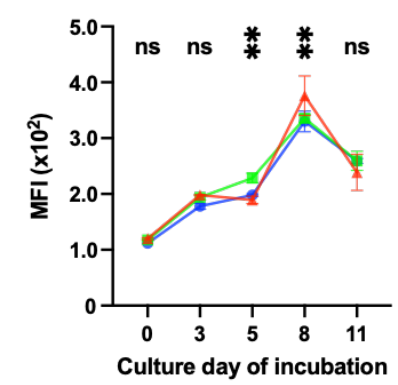

Supplementary Figure 9. Mean fluorescence intensity (MFI) analysis for the delivery of IL2loaded SPION50_7, SPION100_2, and SPION200_2 on the T cells over the course of 12-Day culture. On each indicated day on X-axis, the SPIONs were incubated for 24 hours with the T cells before being analyzed by flow cytometry. " 0 " refers to the unactivated cells. Association (a-c) and uptake (d-f) of SPION50_7 (a, d), SPION100_2 (b, e), and SPION200_2 (c, f) was measured by MFI values and represented as mean $\pm \mathrm{SD}(\mathrm{n}=3$; two-way ANOVA with Dunnett's multiple comparisons, $\left.{ }^{*} p<0.05,{ }^{* *} p=0.0038,{ }^{* * *} p=0.0003,{ }^{* * *} p<0.0001\right)$. 